University of Nebraska - Lincoln

DigitalCommons@University of Nebraska - Lincoln

$11-22-2002$

\title{
Monte Carlo simulation of vapor-liquid binodal of water
}

S. Yoo

University of Nebraska-Lincoln

Xiao Cheng Zeng

University of Nebraska-Lincoln, xzeng1@unl.edu

Follow this and additional works at: https://digitalcommons.unl.edu/chemzeng

Part of the Chemistry Commons

Yoo, S. and Zeng, Xiao Cheng, "Monte Carlo simulation of vapor-liquid binodal of water" (2002). Xiao Cheng Zeng Publications. 39.

https://digitalcommons.unl.edu/chemzeng/39

This Article is brought to you for free and open access by the Published Research - Department of Chemistry at DigitalCommons@University of Nebraska - Lincoln. It has been accepted for inclusion in Xiao Cheng Zeng Publications by an authorized administrator of DigitalCommons@University of Nebraska - Lincoln. 


\title{
LETTERS TO THE EDITOR
}

The Letters to the Editor section is divided into three categories entitled Notes, Comments, and Errata. Letters to the Editor are limited to one and three-fourths journal pages as described in the Announcement in the 1 July 2002 issue.

\section{NOTES}

\section{Monte Carlo simulation of vapor-liquid binodal of water}

\author{
S. Yoo and X. C. Zenga) \\ Department of Chemistry, University of Nebraska-Lincoln, Lincoln, Nebraska 68588
}

(Received 29 July 2002; accepted 30 August 2002)

[DOI: $10.1063 / 1.1516593]$

Among many popular potential models of water, the nonpolarizable four-site TIP4P potential ${ }^{1}$ is one of the more widely used. Recently, two new potential models of water on the basis of the TIP4P potential have been developed. One is the four-site Dang-Chang polarizable potential ${ }^{2}$ and another is the five-site TIP5P potential. ${ }^{3}$ The former is designed to describe not only bulk and interfacial properties of liquid water but also microclusters of water. The nonpolarizable TIP5P potential is the latest version in the TIP series from the Jorgensen group. Compared with the TIP4P potential, for example, the TIP5P potential gives a much improved description of the so-called temperature of maximum density. Recently we used the TIP4P, SPC/E $\mathrm{E}^{4}$ and Dang-Chang potentials to study ion-induced droplet formation. ${ }^{5}$ The supersaturation was not evaluated due to the lack of vapor-liquid coexistence density for the Dang-Chang model. In this note, we report Gibbs ensemble Monte Carlo (GEMC) simulation $^{6,7}$ of vapor-liquid coexistence densities (the binodal curves) for the TIP5P and Dang-Chang models of water. Similar simulations have been carried out for the SPC/E water in the presence of uniform external electric fields. ${ }^{8}$

The molecular parameters for TIP5P and Dang-Chang potentials are listed in Table I. Specifically, the pairwise TIP5P potential is given by the sum of the Lennard-Jones and Coulomb interactions,

$$
U_{\text {pair }}=\sum_{i} \sum_{j>i}\left(4 \epsilon\left[\left(\frac{\sigma}{r_{i j}}\right)^{12}-\left(\frac{\sigma}{r_{i j}}\right)^{6}\right]+\frac{q_{i} q_{j}}{r_{i j}}\right)
$$

where $r_{i j}$ is the distance between site $i$ of one molecule and site $j$ of another molecule; $q_{i}$ is the charge of site $i ; \sigma$ and $\epsilon$ are the Lennard-Jones parameters for the oxygen site. In addition, the Dang-Chang model has a molecular polarizability $\alpha$ of $1.444 \AA{ }^{3}$ The nonadditive polarizable energy of the system $U_{\text {pol }}$ is then given by

$$
U_{\mathrm{pol}}=-\frac{1}{2} \sum_{i} \vec{\mu}_{i} \cdot \vec{E}_{i}^{0}
$$

a)Electronic mail: xzeng1@unl.edu where $\vec{E}_{i}^{0}$ is the electric field produced by all permanent charges in the system at site $i$,

$$
\vec{E}_{i}^{0}=\sum_{j \neq i} \frac{q_{j} \vec{r}_{i j}}{r_{i j}^{3}}
$$

and $\vec{\mu}_{i}$ is the induced dipole at site $i$, which is given by

$$
\vec{\mu}_{i}=\alpha_{i} \vec{E}_{i}=\alpha_{i}\left[\vec{E}_{i}^{0}+\sum_{j \neq i} \mathbf{T}_{i j} \cdot \vec{\mu}_{j}\right],
$$

where $\vec{E}_{i}$ is the total electric field at site $i$, and $\mathbf{T}_{i j}$ is the dipole tensor, i.e.,

$$
\mathbf{T}_{i j}=\frac{1}{r_{i j}^{3}}\left(\frac{3 \vec{r}_{i j} \vec{r}_{i j}}{r_{i j}^{2}}-\mathbf{I}\right),
$$

where $\mathbf{I}$ is the unit tensor. We used a standard iterative selfconsistent field procedure to evaluate the induced dipole with Eq. (4). The iterations were stopped when the maximum difference in the induced dipoles between successive iterations is less than $0.0001 \mathrm{D} / \mathrm{site}$. In all simulations, the water-water interactions were truncated at a molecular separation of $9 \AA$.

To calculate the vapor-liquid coexistence densities (binodal curves), we employed the Gibbs ensemble Monte Carlo simulation method of Panagiotopoulos. ${ }^{6,7}$ In the GEMC method, two simulation cells are used, each representing a portion of one coexisting phase. Three types of MC moves are taken in the simulation: molecular displacement and rotation to assure internal equilibrium; variation of cell volume to satisfy equal pressure conditions; and molecular exchange between two cells to enforce equal chemical potential conditions. We used a 432 water molecule system. We found that this system size is reasonably large to prevent some large density fluctuation due to the molecule exchange. In all simulations, each MC cycle consists of 432 attempts of displacement and rotation, one volume rearrangement attempt, and one molecule exchange attempt. The number of MC cycles in the equilibration run typically ranged from 100000 to 1000000 to assure the system is in the equilibrium state. After the equilibration run, another 1000000 cycles were then used for data collection in the case of the TIP5P model. In the case of the Dang-Chang model, 100000 cycles were 
TABLE I. Molecular parameters for TIP5P and Dang-Chang water models. The TIP5P water model has two negative charge sites (denoted M) located at two lone-pair interaction sites. The Dang-Chang model has one negative charge site which is shifted from the position of the oxygen site (the Lennard-Jones site) by $r_{\mathrm{OM}}$ toward the direction of the permanent dipole. $\alpha$ is the molecular polarizability.

\begin{tabular}{ccc}
\hline \hline & TIP5P & Dang-Chang \\
\hline$r_{\mathrm{OH}}(\AA)$ & 0.9572 & 0.9572 \\
$r_{\mathrm{OM}}(\AA)$ & 0.70 & 0.215 \\
$\theta_{\mathrm{HOH}}(\mathrm{deg})$ & 104.52 & 104.52 \\
$\theta_{\mathrm{MOM}}(\mathrm{deg})$ & 109.47 & \\
$\epsilon_{\mathrm{LJ}}(\mathrm{kcal} / \mathrm{mol})$ & 0.16 & 0.1825 \\
$\sigma_{\mathrm{LJ}}(\AA)$ & 3.12 & 3.234 \\
$q_{\mathrm{H}}(e)$ & 0.241 & 0.519 \\
$q_{\mathrm{M}}(e)$ & -0.241 & -1.038 \\
$\alpha\left(\AA^{3}\right)$ & & 1.44 \\
\hline \hline
\end{tabular}

used due to the much longer computational time needed for the polarizable model. The simulation results are shown in Table II.

Because the critical point cannot be determined directly from the GEMC simulation, we fitted the vapor-liquid coexistence densities $\left(\rho_{v}\right.$ and $\left.\rho_{l}\right)$, except those of $298.15 \mathrm{~K}$, to the scaling law

$$
\rho_{l}-\rho_{v}=A\left(T_{c}-T\right)^{\beta}
$$

and the rectilinear law,

$$
\frac{\rho_{l}+\rho_{v}}{2}=\rho_{c}+B\left(T_{c}-T\right),
$$

where $T_{c}$ is the critical temperature, $\rho_{c}$ is the critical density, and $A$ and $B$ are two associated coefficients. For threedimensional systems, the critical exponent $\beta$ is 0.326 . The final estimated $T_{c}$ is 546 and $590 \mathrm{~K}$ for the TIP5P and the Dang-Chang model water, respectively. ${ }^{10}$ Note that both values of $T_{c}$ are lower than the experimental value of $647 \mathrm{~K}$. $^{11}$

In Fig. 1, we plot the binodal curves of the TIP5P and Dang-Chang model water, along with the binodal curves of TIP4P and SPC/E model water (Ref. 9). Compared with the TIP4P model $\left(T_{c}=579 \mathrm{~K}\right)$, the TIP5P model gives a lower $T_{c}$ of $546 \mathrm{~K}$ whereas the Dang-Chang model yields a higher $T_{c}(590 \mathrm{~K})$. It is known that a fluid with a stronger dipole

TABLE II. Vapor-liquid coexistence densities of TIP5P and Dang-Chang model water. Numbers in parentheses denote the error of the simulation data.

\begin{tabular}{cccccc}
\hline \hline & \multicolumn{3}{c}{ TIP5P } & & \multicolumn{2}{c}{ Dang-Chang } \\
\cline { 2 - 3 } \cline { 5 - 6 }$T(\mathrm{~K})$ & $\rho_{g}\left(\mathrm{~g} / \mathrm{cm}^{3}\right)$ & $\rho_{l}\left(\mathrm{~g} / \mathrm{cm}^{3}\right)$ & & $\rho_{g}\left(\mathrm{~g} / \mathrm{cm}^{3}\right)$ & $\rho_{l}\left(\mathrm{~g} / \mathrm{cm}^{3}\right)$ \\
\hline 298.15 & $0.0000549(11)$ & $0.989(4)$ & & $0.0000488(4)$ & $0.997(13)$ \\
323 & $0.000221(4)$ & $0.970(3)$ & & $0.000219(2)$ & $0.967(6)$ \\
373 & $0.00148(4)$ & $0.908(3)$ & & $0.00149(1)$ & $0.917(11)$ \\
423 & $0.00611(27)$ & $0.824(3)$ & & $0.00797(24)$ & $0.851(7)$ \\
473 & $0.0241(12)$ & $0.711(6)$ & & $0.0249(13)$ & $0.764(8)$ \\
503 & $0.0564(8)$ & $0.622(13)$ & & \\
\hline \hline
\end{tabular}

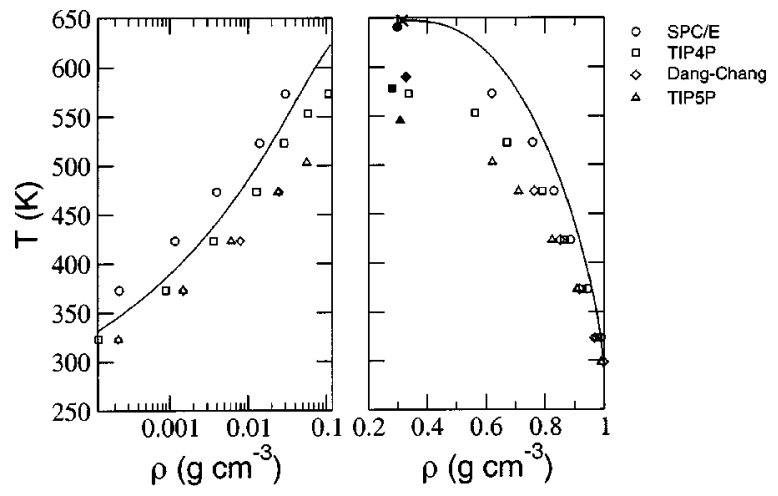

FIG. 1. Vapor-liquid binodal curves of TIP5P and Dang-Chang model water. Simulation results are compared with those of TIP4P and SPC/E model water. The solid line represents experimental values (Ref. 11). The open circles, squares, diamonds, and triangles are simulation results of SPC/E, TIP4P, Dang-Chang, and TIP5P model, respectively (Ref. 9). The closed symbols represent the estimated critical point for each water model by using Eqs. (6) and (7). The cross denotes the measured critical point of water from experiments.

moment tends to have a higher $T_{c}$. Indeed, the polarizable Dang-Chang model has effectively stronger dipole moment (permanent plus induced dipole) $)^{2}$ than that of TIP4P $(2.18$ D). On the other hand, the dipole moment of TIP5P model (2.29 D) is slightly higher than TIP4P and yet the $T_{c}$ of TIP5P water is lower than that of TIP4P. This apparently peculiar behavior can be understood on the basis ${ }^{12}$ that the five-site TIP5P model has much smaller quadrupole moments $Q_{x x}$ and $Q_{y y}\left(1.65 \times\right.$ and $-1.48 \times 10^{-26}$ esu cm ${ }^{2}$, respectively) in magnitude than those of TIP4P $(2.20 \times$ and $-2.09 \times 10^{-26} \mathrm{esu} \mathrm{cm}^{2}$ ) as well as those of the Dang-Chang model $\left(2.24 \times\right.$ and $\left.-2.05 \times 10^{-26} \mathrm{esu} \mathrm{cm}^{2}\right)$.

We thank Dr. Liem Dang for sending us simulation data of Ref. 2 and Dr. G.T. Gao and J. Bai for valuable discussions. This work is supported by the National Science Foundation (X.C.Z.).

${ }^{1}$ W. L. Jorgensen, J. Chandresekhar, J. D. Madura, R. W. Impey, and M. L. Klein, J. Chem. Phys. 79, 926 (1983).

${ }^{2}$ L. X. Dang and T.-M. Chang, J. Chem. Phys. 106, 8149 (1997).

${ }^{3}$ M. W. Mahoney and W. L. Jorgensen, J. Chem. Phys. 112, 8910 (2000).

${ }^{4}$ H. J. C. Berendsen, J. R. Grigera, and T. P. Straatsma, J. Phys. Chem. 91, 6269 (1987).

${ }^{5}$ K. J. Oh, G. T. Gao, and X. C. Zeng, Phys. Rev. Lett. 86, 5080 (2001).

${ }^{6}$ A. Z. Panagiotopoulos, Mol. Phys. 61, 813 (1987).

${ }^{7}$ A. Z. Panagiotopoulos, N. Quirke, M. R. Stapleton, and D. J. Tildesley, Mol. Phys. 63, 527 (1988).

${ }^{8}$ G. T. Gao, K. J. Oh, and X. C. Zeng, J. Chem. Phys. 110, 2533 (1999).

${ }^{9}$ J. Vorholz, V. I. Harismiadis, B. Rumpf, A. Z. Panagiotopoulos, and G. Maurer, Fluid Phase Equilibria 170, 203 (2000).

${ }^{10} T_{c}$ will be less than 546 and $590 \mathrm{~K}$ (for TIPSP and Dang-Chang model) if the Ewald sum method is used to account for long-range interactions (private communications with L. X. Dang and B. Chen).

${ }^{11}$ E. Schmidt, Properties of Water and Steam in SI-Unit (Springer, Berlin, 1969).

${ }^{12}$ V. B. Warshavsky and X. C. Zeng, J. Chem. Phys. 117, 3982 (2002). 